PROCEEDINGS OF THE

AMERICAN MATHEMATICAL SOCIETY

Volume 139, Number 3, March 2011, Pages 1033-1044

S 0002-9939(2010)10517-8

Article electronically published on August 2, 2010

\title{
CRITICAL TYPE OF KRASNOSEL'SKII FIXED POINT THEOREM
}

\author{
TIAN XIANG AND RONG YUAN
}

(Communicated by Yingfei Yi)

\begin{abstract}
In this paper, by means of the technique of measures of noncompactness, we establish a generalized form of the fixed point theorem for the sum of $T+S$, where $S$ is noncompact, $I-T$ may not be injective, and $T$ is not necessarily continuous. The obtained results unify and significantly extend a number of previously known generalizations of the Krasnosel'skii fixed point theorem. The analysis presented here reveals the essential characteristics of the Krasnosel'skii type fixed point theorem in strong topology setups. Further, the results are used to prove the existence of periodic solutions of a nonlinear neutral differential equation with delay in the critical case.
\end{abstract}

\section{INTRODUCTION}

It is well known that the Krasnosel'skii fixed point theorem is a combination of the Banach contraction mapping principle and the Schauder fixed point theorem. The theorem was motivated by an observation that the inversion of a perturbed differential operator may yield the sum of a compact and a contraction operator. The sum of operators frequently appears in delay integral equations and neutral functional equations, which have been studied widely in [8, 15], for instance. Krasnosel'skii stated that the sum $T+S$ has at least one fixed point in a nonempty, closed convex subset $K$ of a Banach space $E$, where $S$ and $T$ fulfill:

(i) $T$ is a contraction with constant $\alpha<1$;

(ii) $S$ is continuous and $S(K)$ resides in a compact subset of $E$;

(iii) any $x, y \in K$ imply $T x+S y \in K$.

The theorem is useful in establishing the existence results for perturbed operator equations. It also has a wide range of applications to nonlinear integral equations of mixed type for proving the existence of solutions. Thus the existence of fixed points for the sum of two operators has attracted tremendous interest, and their applications are frequent in nonlinear analysis. Many improvements of the theorem have been established in the literature in the course of time by modifying the assumptions (i), (ii) or (iii); see 4, 7, 16. It was mentioned in 4 that the condition (iii) is too stringent and can be replaced by a mild one. Burton proposed the following improvement for (iii): if $x=T x+S y$ with $y \in K$, then $x \in K$, and

Received by the editors March 14, 2009 and, in revised form, March 29, 2010.

2010 Mathematics Subject Classification. Primary 47H08, 47H10, 37C25.

Key words and phrases. Fixed point, noncompact mapping, multi-valued mapping.

This work was supported by National Natural Science Foundation of China.

(C)2010 American Mathematical Society Reverts to public domain 28 years from publication 
an important variant of the theorem was obtained. Subsequently, in [7, Dhage replaced (i) by the following requirement: $T$ is a bounded linear operator on $E$, and $T^{p}$ is a nonlinear contraction for some $p \in \mathbb{N}$. More recently, in [16], the authors first replaced the contractive map by an expansive one and then replaced the compactness of the operator $S$ by a $k$-set contractive one, and obtained some new fixed results, which extend and develop some previously related fixed results.

Up to now there have appeared many other fixed point theorems for the sum of two operators, their generalizations and variants of Krasnosel'skii's fixed point theorem; for example, see [4, 5, 7, 11, 12, 14, 16] and the references therein. It is well known, on the one hand, in some previous related works, that the compactness of $S$ plays a crucial role in their arguments. The possible reason for this is that their discussions are based upon Schauder's fixed point theorem. On the other hand, in most of the above quoted works, the assumptions that $T$ is continuous and $I-T$ is continuously invertible also play a fundamental role in their arguments. However, in some kinds of applications, one may encounter the case that $I-T$ is not injective or/and $T$ is not continuous. So it is necessary to further study the problem in the absence of the above-described properties for the sake of theoretical interest and practical application. Thus it would be interesting to study the case when $I-T$ is not injective and $T$ is not continuous. It is the purpose of this paper that we continue to explore this kind of extension by looking for the resulting mapping $(I-T)^{-1} S$ attaining a fixed point in some subset of $K$. The arguments developed here disclose the essential characteristics of the Krasnosel'skii type fixed point theorem in strong topology settings. By means of the technique of measures of noncompactness, we do not necessarily require that $S$ is compact and that $T$ is continuous. Indeed, the continuity assumption of $T$ is substantially lessened (see Remark 2.2). It should be mentioned that other authors have already investigated such types of problems. For instance, in [11, the authors assume that $S$ is compact and $T$ is continuous; in [12, the author considered the fixed set of Krasnosel'skii type for the case when $S$ is a compact and continuous multi-valued mapping. It is shown (see Remarks 2.2 and 2.3) that our results largely generalize and encompass a number of existing generalizations or modifications of the Krasnosel'skii fixed point theorem. Finally, our results are used to prove some existence principles for a nonlinear neutral differential equation with delay in the critical case.

For the remainder of this section, we collect some definitions and recall some basic facts that are used further on. Let $E$ be a Banach space and $M \subset E$; set

$$
P(M)=\{N: N \subset M, N \neq \emptyset\} .
$$

A multi-valued mapping $F: M \rightarrow P(E)$ is said to be

(1) upper semi-continuous if for each closed set $A \subset E, F^{-1}(A)=\{x \in E$ : $F(x) \cap A \neq \emptyset\}$ is closed in $E$;

(2) closed if its graph $G(F)=\{(x, y) \in M \times E: y \in F(x)\}$ is closed;

(3) compact if $F(M)=\bigcup_{x \in M} F(x)$ is a relatively compact subset of $E$.

The following result plays the role of a prototype in our discussion of this paper.

Theorem A ([17, p. 452]). Let $M$ be a closed and convex subset of a Banach space $E$ and $F: M \rightarrow P(M)$ a multi-valued mapping. Suppose that

(i) F is a compact multi-valued mapping; 
(ii) $F$ is upper semi-continuous on $M$;

(iii) the set $F(x)$ is closed and convex for all $x \in M$.

Then there exists $x \in M$ such that $x \in F(x)$.

\section{CRitical tyPe of Krasnosel'Skit FiXed POINT THEOREM}

For a bounded set $A$ in $E$, the Kuratowskii measure of noncompactness $\alpha_{E}(A)$ (simply $\alpha(A))$ is defined by

$$
\begin{array}{r}
\alpha_{E}(A)=\inf \left\{\delta>0 \mid \quad \text { there is a finite number of subsets } A_{i} \subset A\right. \\
\text { such that } \left.A \subseteq \bigcup_{i} A_{i} \text { and } \operatorname{diam}\left(A_{i}\right) \leq \delta\right\} .
\end{array}
$$

The Hausdorff measure of non-compactness $\gamma_{E}(A)$ (simply $\left.\gamma(A)\right)$ is defined by

$$
\gamma_{E}(A)=\inf \{\epsilon>0 \mid A \text { admits a finite } \epsilon \text {-net in } E\}
$$

In this paper we need mainly the fact that $A \subset E$ is relatively compact if and only if $\psi(A)=0$, where and henceforth $\psi$ stands for either $\alpha$ or $\gamma$. For the other properties of $\alpha$ and $\gamma$ we refer to [1, 2. By using the techniques of measures of noncompactness, for our further purpose we first remove the compactness of $F$ in Theorem $\mathrm{A}$ in the case that $M$ is bounded.

Theorem 2.1. Let $M$ be a bounded, closed and convex subset of $E$ and $F: M \rightarrow$ $P(M)$ a multi-valued mapping. Suppose that

(i) $\psi(F(A))<\psi(A)$ for all $A \subset M$ with $\psi(A)>0$;

(ii) $F$ is upper semi-continuous on $M$;

(iii) the set $F(x)$ is closed and convex for all $x \in M$.

Then there exists $x \in M$ such that $x \in F(x)$.

Proof. Let $x_{0} \in M$ and

$$
\mathcal{A}=\left\{A: x_{0} \in A \subset M, A \text { is a closed convex set and } F: A \rightarrow P(A)\right\} .
$$

Clearly, $\mathcal{A} \neq \emptyset$ since $M \in \mathcal{A}$. Moreover, put $A_{0}=\bigcap_{A \in \mathcal{A}} A$. Then $x_{0} \in A_{0} \subset M, A_{0}$ is also a closed convex set and $F: A_{0} \rightarrow P\left(A_{0}\right)$. Notice that $\overline{c o}\left\{F\left(A_{0}\right), x_{0}\right\} \subset A_{0}$, where $\overline{c o}(A)$ denotes the closed convex hull of the set $A$. Thus, we have

$$
F\left(\overline{c o}\left\{F\left(A_{0}\right), x_{0}\right\}\right) \subset F\left(A_{0}\right) \subset \overline{c o}\left\{F\left(A_{0}\right), x_{0}\right\},
$$

which shows that $\overline{c o}\left\{F\left(A_{0}\right), x_{0}\right\} \in \mathcal{A}$. It then follows that $\overline{c o}\left\{F\left(A_{0}\right), x_{0}\right\}=A_{0}$. Hence,

$$
\psi\left(A_{0}\right)=\psi\left(\overline{c o}\left\{F\left(A_{0}\right), x_{0}\right\}\right)=\psi\left(\left\{F\left(A_{0}\right), x_{0}\right\}\right)=\psi\left(F\left(A_{0}\right)\right) .
$$

Note that $A_{0} \subset M$ and $M$ is bounded; thus $\psi\left(A_{0}\right)$ is well-defined. The above equality together with (i) now implies that $\psi\left(A_{0}\right)=0$. Therefore, one concludes that $A_{0}$ is a nonempty compact convex subset of $M$ and $F: A_{0} \rightarrow P\left(A_{0}\right)$. Now, applying Theorem $\mathrm{A}$ to $F$ on $A_{0}$, one obtains that there exists $x \in A_{0}$ such that $x \in F(x)$. This accomplishes the proof.

Remark 2.1. When $F$ is a single-valued condensing mapping, Theorem 2.1 is known as the classical Sadovskii fixed point theorem. 
Let $M, K$ be two subsets of $E, T: M \rightarrow E$ and $S: K \rightarrow E$ two mappings. We shall denote by $\mathcal{F}=\mathcal{F}(M, K ; T, S)$ the following set:

$$
\mathcal{F}=\{x \in M: x=T x+S y \text { for some } y \in K\} .
$$

We are now in a position to employ Theorem A or Theorem 2.1 to deduce the following critical type of the Krasnosel'skii fixed point theorem; by saying "critical type" we mean that $I-T$ can be allowed to be noninvertible.

Theorem 2.2. Let $K \subset E$ be a nonempty, bounded, closed and convex subset and $T: E \rightarrow E$ a map. Suppose that $S: K \rightarrow E$ is continuous such that

(i) $S(K) \subset(I-T)(E)$ and $[x=T x+S y, y \in K] \Longrightarrow x \in K$ (or $S(K) \subset$ $(I-T)(K))$

(ii) $\psi(T(A)+S(A))<\psi(A)$ for all $A \subset K$ with $\psi(A)>0$;

(iii) if $\left\{x_{n}\right\}$ is a sequence in $\mathcal{F}(E, K ; T, S)$ and $(I-T) x_{n} \rightarrow y$, then $\left\{x_{n_{k}}\right\}$ is convergent, where $\left\{x_{n_{k}}\right\}$ is a subsequence of $\left\{x_{n}\right\}$;

(iv) $T$ is closed in $\mathcal{F}$; that is, if $\left\{x_{n}\right\} \subset \mathcal{F}$ for which $x_{n} \rightarrow x$ and $T x_{n} \rightarrow y$, then $y=T x$;

(v) for each $y$ in the range of $S, \Delta_{y}=\{x \in E:(I-T) x=y\}$ is a convex set. Then there exists a point $x^{*} \in K$ with $S x^{*}+T x^{*}=x^{*}$.

Proof. From the first part of (i), for each $x \in K$, we see that there exists $y \in E$ such that

$$
T y+S x=y .
$$

We first assume that $I-T$ is invertible. This together with the second part of (i) yields that $y=(I-T)^{-1} S x:=N x \in K$ for each $x \in K$. Therefore, one obtains that $N$ maps $K$ into itself. Let $x_{0} \in K$ and

$$
\mathcal{A}=\left\{A: x_{0} \in A \subset K, A \text { is a closed convex set and } N(A) \subset A\right\} .
$$

Clearly, $\mathcal{A} \neq \emptyset$ since $K \in \mathcal{A}$. Moreover, for any $A \in \mathcal{A}$ we have that

$$
(I-T)^{-1} S(A) \subset T(I-T)^{-1} S(A)+S(A) \subset T(A)+S(A) .
$$

It follows from (2.2) and (ii) that

$$
\psi(N(A))<\psi(A) \text { for all } A \in \mathcal{A} \text { with } \psi(A)>0 .
$$

Put $A_{0}=\bigcap_{A \in \mathcal{A}} A$. Then $x_{0} \in A_{0} \subset K, A_{0}$ is also a closed convex set and $N\left(A_{0}\right) \subset A_{0}$. Notice that $\overline{c o}\left\{N\left(A_{0}\right), x_{0}\right\} \subset A_{0}$. Thus, we have

$$
N\left(\overline{c o}\left\{N\left(A_{0}\right), x_{0}\right\}\right) \subset N\left(A_{0}\right) \subset \overline{c o}\left\{N\left(A_{0}\right), x_{0}\right\},
$$

which shows that $\overline{c o}\left\{N\left(A_{0}\right), x_{0}\right\} \in \mathcal{A}$. It follows that $\overline{c o}\left\{N\left(A_{0}\right), x_{0}\right\}=A_{0}$. Hence,

$$
\psi\left(A_{0}\right)=\psi\left(\overline{c o}\left\{N\left(A_{0}\right), x_{0}\right\}\right)=\psi\left(\left\{N\left(A_{0}\right), x_{0}\right\}\right)=\psi\left(N\left(A_{0}\right)\right) .
$$

It follows from (2.3) and (2.4) that $\psi\left(A_{0}\right)=0$. Therefore, $A_{0}$ is a nonempty compact convex subset of $K$ and $N\left(A_{0}\right) \subset A_{0}$. We next claim that $N: A_{0} \rightarrow A_{0}$ is continuous. Indeed, let $\left\{x_{n}\right\}$ be a sequence in $A_{0}$ with $x_{n} \rightarrow x$ in $A_{0}$. Set $y_{n}=(I-T)^{-1} S x_{n}$ and $y=(I-T)^{-1} S x$. Then $(I-T) y_{n}=S x_{n}$ and $(I-T) y=S x$. Hence $y_{n}, y \in A_{0} \cap \mathcal{F}$ and

$$
(I-T) y_{n} \rightarrow(I-T) y
$$


One derives from (2.5) and (iii) that $y_{n_{k}} \rightarrow y_{0}$ for some $y_{0} \in A_{0}$, where $\left\{y_{n_{k}}\right\}$ is a subsequence of $\left\{y_{n}\right\}$. We then deduce from (2.5) that

$$
T y_{n_{k}} \rightarrow y_{0}-(I-T) y .
$$

It follows from (2.6) and (iv) that $y_{0}-(I-T) y=T y_{0}$, and so $y_{0}=y$ since $I-T$ is injective. This in turn shows that $y_{n} \rightarrow y$. The claim is therefore proved.

Now the Schauder fixed point theorem implies that $N=(I-T)^{-1} S$ has at least one fixed point in $A_{0}$. In such a case, we are through.

If $I-T$ is not invertible, then $(I-T)^{-1}$ could be viewed as a multi-valued mapping. For each given $x \in K$, define $F: K \rightarrow P(K)$ by $F x=(I-T)^{-1} S x$. Then $F$ is well defined by condition (i). Since $H \circ H^{-1}(A) \subset A$ for any mapping $H$, we discover that (2.2) is also valid in such a case. Consequently, repeating the above discussion or employing Theorem 2.1, one derives that there exists a nonempty compact convex subset $A_{0}$ of $K$ such that $F: A_{0} \rightarrow P\left(A_{0}\right)$. We next illustrate that $F$ satisfies all the requirements of Theorem A or Theorem 2.1 on the set $A_{0}$.

Step 1. $F\left(A_{0}\right)$ is relatively compact. For any $\left\{x_{n}\right\} \subset F\left(A_{0}\right)$, we choose $\left\{y_{n}\right\} \subset$ $A_{0}$ such that $x_{n} \in F\left(y_{n}\right)$. By the definition of $F$, one has $(I-T) x_{n}=S y_{n}$. Since $S\left(A_{0}\right)$ is relatively compact, we may assume that $\left\{(I-T) x_{n}\right\}$ converges to $z$ for some $z \in E$. Taking (iii) into account, one knows that there exists a subsequence $\left\{x_{n_{k}}\right\}$ of $\left\{x_{n}\right\}$ converging to $x_{0}$ for some $x_{0} \in A_{0}$.

Step 2. $F(x)$ is a convex set for each $x \in A_{0}$. This is an immediate consequence of assumption (v).

Step 3. $F$ is a closed multi-valued mapping on $A_{0}$. Let $\left\{x_{n}\right\} \subset A_{0}$ with $x_{n} \rightarrow x$, and let $y_{n} \in F\left(x_{n}\right)$ with $y_{n} \rightarrow y$. Then one has $x \in A_{0}$ and $(I-T) y_{n}=S x_{n}$. This shows that $(I-T) y_{n} \rightarrow S x$ because $S$ is continuous in $K\left(\supset A_{0}\right)$. It further implies that $T y_{n} \rightarrow y-S x$. We then conclude by (iv) that $y-S x=T y$, and so $y \in(I-T)^{-1} S x$. This checks that the graph $G(F)$ is closed, i.e., that $F$ is a closed multi-valued mapping on $A_{0}$.

Step 4. $F(x)$ is a nonempty closed set for each $x \in A_{0}$. This assertion follows from the first part of condition (i) and Step 3.

One may infer from Steps 1 and 3 that $F$ is upper semi-continuous on $A_{0}$. By invoking Theorem A or Theorem 2.1] one obtains that there exists $x^{*} \in A_{0}$ such that $x^{*} \in F\left(x^{*}\right)$. Accordingly, $S x^{*}+T x^{*}=x^{*}$. This completes the proof.

Remark 2.2. From the proof of Theorem 2.2, it is readily seen that one of the advantages of Theorem 2.2 is that instead of requiring that $(I-T)^{-1}$ be continuous in its domain we require only that $(I-T)^{-1} S: A_{0} \rightarrow A_{0}$ be continuous. Another advantage is that the compactness of $S$ is relaxed. The method developed here exposes the essential characteristics of the Krasnosel'skii type fixed point in strong topology circumstances; that is, by imposing suitable conditions on the operators $S$ and $T$, we are then able to find a possible smallest nonempty compact convex set $A_{0}$, so that either the Schauder fixed point principle or its multi-value version, Theorem A, is applicable to the resulting mapping $N=(I-T)^{-1} S$ on the set $A_{0}$. We also make the following useful observations.

1. It is obvious that the condition (iv) is much weaker than the condition that $T$ is continuous. Clearly, if $T$ is continuous, then it is closed; conversely 
this may not be true. The condition (ii) can be replaced by

(ii') $S(K)$ resides in a compact subset of $E$.

In such a case, it can be shown by the properties of measures of noncompactness that $\psi(T(A)+S(A))=\psi(T(A))$ for all $A \subset K$ with $\psi(A)>0$; moreover, the boundedness of $K$ is not required; see Remark 2.3. Thus the compactness assumption of $S$ and the continuity assumption of $T$, both of which are required in [11, are relaxed.

2. If $(I-T)^{-1}$ is continuous, then the hypotheses (iii) and (v) are fulfilled, and the condition (iv) is redundant. This implies that Theorem 2.2 is valid regardless of the continuity of $T$. Furthermore, in such a case, we can impose conditions on $(I-T)^{-1}$ and $S$ so that $\psi\left((I-T)^{-1} S(A)\right)<\psi(A)$ for all $A \subset K$ with $\psi(A)>0$ instead of (ii). For example, we can assume that $S$ is $k$-set condensing and $(I-T)^{-1}$ is $k^{-1}$-set contractive instead of requiring that (ii) holds [16. The assumption that $I-T$ is continuous and invertible (continuously invertible) has been studied widely in [4, 5, 17, 14, 16.

3. If $I-T$ is continuously invertible and $T$ is continuous, then the assumptions (iii)-(v) are satisfied. In particular, if $T$ is a contraction (or a nonlinear contraction or a large contraction [11) on $E$, then the assumptions (iii)-(v) are satisfied. Furthermore it can be shown that $(I-T)(E)=E$ and hence the first part of (i) is also fulfilled. Accordingly Theorem 2.2 generalizes Theorem 2.11 in [16], and a number of previously known generalizations or modifications of the Krasnosel'skii fixed point theorem 4, 5, 7, 14.

4. The condition (v) is satisfied provided that $T$ is convex; that is, $T(\alpha x+$ $\beta y)=\alpha T x+\beta T y$ for all $x, y \in E$ and all $\alpha, \beta \geq 0$ with $\alpha+\beta=1$. In particular, Theorem 2.2 may facilitate the application of another kind of operator, namely, a compact linear operator, for the Fredholm alternative principle tells us that $\operatorname{ker}(I-T)=\{\theta\}$ if and only if $(I-T)(E)=E$ and that $(I-T)(E)=\operatorname{ker}\left(I^{*}-T^{*}\right)^{\perp}$. These benefits will enable the assumptions to be amiable, especially in Hilbert spaces.

Let us now shift our attention to the case when $K$ is not necessarily bounded. By imposing an appropriate compactness assumption on the set $\mathcal{F}$, we shall derive an abstract strong type Krasnosel'skii fixed point theorem, which unifies and substantially extends a multitude of previously known related results.

Theorem 2.3. Let $K \subset E$ be a nonempty, closed and convex subset and $T: E \rightarrow E$ a map. Suppose that $S: K \rightarrow E$ is continuous such that

(i) $S(K) \subset(I-T)(E)$ and $[x=T x+S y, y \in K] \Longrightarrow x \in K$ (or $S(K) \subset$ $(I-T)(K))$

(ii) the set $\mathcal{F}(E, K ; T, S)$ is relatively compact;

(iii) if $\left\{x_{n}\right\}$ is a sequence in $\mathcal{F}(E, K ; T, S)$ and $(I-T) x_{n} \rightarrow y$, then $\left\{x_{n_{k}}\right\}$ is convergent, where $\left\{x_{n_{k}}\right\}$ is a subsequence of $\left\{x_{n}\right\}$;

(iv) if $\left\{x_{n}\right\} \subset \mathcal{F}$ for which $x_{n} \rightarrow x$ and $T x_{n} \rightarrow y$, then $y=T x$;

(v) for each $y$ in the range of $(I-T), \Delta_{y}=\{x \in E:(I-T) x=y\}$ is a convex set.

Then there exists a point $x^{*} \in K$ with $S x^{*}+T x^{*}=x^{*}$.

Proof. It is sufficient to demonstrate, when $I-T$ is one-to-one, that the resulting mapping $N:=(I-T)^{-1} S: K \rightarrow K$ is compact and continuous. In fact, let 
$y_{n} \in N(K)$. Then $(I-T) y_{n}=S x_{n}$ for some $x_{n} \in K$ and hence $y_{n} \in \mathcal{F}$ by the definition of $\mathcal{F}$. Keeping assumption (ii) in mind, one obtains that $\left\{y_{n}\right\}$ possesses a convergent subsequence. Now let $\left\{x_{n}\right\} \subset K$ with $x_{n} \rightarrow x$ in $K$. Put $y_{n}=N x_{n}$ and $y=N x$. Then $y_{n} \in \mathcal{F}$ and $(I-T) y_{n} \rightarrow S x$. One knows from (iii) that $y_{n_{k}} \rightarrow y_{0}$ for some $y_{0} \in K$, where $\left\{y_{n_{k}}\right\}$ is a subsequence of $\left\{y_{n}\right\}$. Consequently, one deduces as before that $y_{0}=N x=y$ and $y_{n} \rightarrow y$. This verifies our claim. The remainder of the proof follows along the lines of Theorem 2.2 .

Remark 2.3. In [11, the authors suppose that $T$ is continuous and

(iii') if $(I-T) x_{n} \rightarrow y$, then there exists a convergent subsequence $\left\{x_{n_{k}}\right\}$ of $\left\{x_{n}\right\}$; and then they claim that, when $I-T$ is invertible, it is easy to see that $(I-T)^{-1}$ is continuous. However, this assertion seems not to be said so easily and may not be correct. An alternative remedy to ensure that the assertion holds true may be to replace condition (iii') by (iii).

It can be readily known from (i) that $\mathcal{F}(E, K ; T, S)=(I-T)^{-1}(K)$. Therefore $\mathcal{F}$ is precompact provided $S(K)$ is. Indeed, let $x_{n} \in \mathcal{F}$. Then $(I-T) x_{n}=S y_{n}$ for some $y_{n} \in K$, and so $(I-T) x_{n_{k}} \rightarrow z$ for some $z \in E$. It then follows from (iii) that $\left\{x_{n}\right\}$ has a convergent subsequence.

Since the continuity of $T$ is largely weakened and the compactness hypothesis of $S$ is placed well, Theorem 2.3 may be seen as a significant improvement of Theorem 2.1 in 11]; it also may be essentially viewed as an extension of the preceding theorem.

\section{Periodic SOlutions For Delay differential EQUations}

Let $C_{2 \pi}=\{x \in C(\mathbb{R}, \mathbb{R}) \mid x(t+2 \pi)=x(t), t \in \mathbb{R}\}$ be a Banach space equipped with the supremum norm

$$
\|x\|=\sup _{t \in \mathbb{R}}|x(t)|=\max _{t \in[0,2 \pi]}|x(t)| .
$$

Let us investigate a kind of biological model. When modeled under a mathematical viewpoint, it is the following nonlinear neutral differential equation with constant delay:

$$
\frac{d}{d t}[x(t)-a x(t-\tau)]=r(t) x(t)-f(t, x(t-\tau)),
$$

where $f: \mathbb{R} \times \mathbb{R} \rightarrow \mathbb{R}$ is a continuous function, $f(t+2 \pi, x)=f(t, x)$ for all $t, x \in \mathbb{R}$, $r \in C_{2 \pi}, a \in \mathbb{R}$ and $\tau \in \mathbb{R}$ are constants.

The system (3.1) arises in food-limited population models and blood cell models (see 3, 10, for example). For a historical background and discussion of applications of (3.1) to a number of dynamical models, we refer the interested investigator to 6 , 13 and the references therein. For system (3.1), there may be a stable equilibrium point of the population. In the case that the equilibrium point becomes unstable, there may exist a nontrivial periodic solution. Then the oscillation of solutions occurs. The existence of such a stable periodic solution is of quite significance biologically because it concerns the long time survival of the species. The study of such phenomena has become an important part of the qualitative theory of differential equations.

In this section, we shall apply Theorem 2.2 or Theorem 2.3 to study the existence of periodic solutions for the system (3.1). In the sequel, we will denote by $\bar{r}$ the 
integral average notation $\int_{0}^{2 \pi} r(s) d s$. Let us begin with the following "bridge" lemma.

Lemma 3.1. Assume $\bar{r} \neq 0$. Then $x \in C_{2 \pi}$ is a solution of system (3.1) if and only if

$$
x(t)=a x(t-\tau)+\int_{t}^{t+2 \pi} G(t, s)[f(s, x(s-\tau))-\operatorname{ar}(s) x(s-\tau)] d s,
$$

where

$$
G(t, s)=\frac{e^{\int_{s}^{t} r(u) d u}}{1-e^{-\int_{0}^{2 \pi} r(u) d u}} .
$$

Proof. Let $x(t) \in C_{2 \pi}$ be a solution of (3.1). We rewrite it in the form

$$
\frac{d}{d t}[x(t)-a x(t-\tau)]=r(t)[x(t)-a x(t-\tau)]-[f(t, x(t-\tau))-\operatorname{ar}(t) x(t-\tau)] .
$$

We next multiply equation (3.4) by $e^{-\int_{0}^{t} r(u) d u}$ and then integrate from $t$ to $t+2 \pi$ to discover

$$
\begin{aligned}
& {[x(t+2 \pi)-a x(t+2 \pi-\tau)] e^{-\int_{0}^{t+2 \pi} r(u) d u}-[x(t)-a x(t-\tau)] e^{-\int_{0}^{t} r(u) d u}} \\
& =-\int_{t}^{t+2 \pi}[f(s, x(s-\tau))-\operatorname{ar}(s) x(s-\tau)] e^{-\int_{0}^{s} r(u) d u} d s .
\end{aligned}
$$

Recalling that $x(t+2 \pi)=x(t)$ and $\bar{r} \neq 0$, the above expression can be put in the form

$$
x(t)=a x(t-\tau)+\int_{t}^{t+2 \pi} \frac{e^{\int_{s}^{t} r(u) d u}}{1-e^{-\int_{0}^{2 \pi} r(u) d u}}[f(s, x(s-\tau))-\operatorname{ar}(s) x(s-\tau)] d s,
$$

and the proof is complete.

In view of Lemma 3.1, the original problem can be transformed into a fixed point one. To do this, we shall construct two mappings:

$$
(T x)(t)=a x(t-\tau), \quad t \in \mathbb{R},
$$

and

$$
(S y)(t)=\int_{t}^{t+2 \pi} G(t, s)[f(s, y(s-\tau))-\operatorname{ar}(s) y(s-\tau)] d s, \quad t \in \mathbb{R} .
$$

It is obvious that $T$ maps $C_{2 \pi}$ into itself. It follows from (3.3) that $G(t+2 \pi, s+2 \pi)=$ $G(t, s)$ for all $t, s \in \mathbb{R}$. Then a change of variable yields that $(S y)(t+2 \pi)=(S y)(t)$ for all $t \in \mathbb{R}$.

Lemma 3.2. Assume that $z, \mu \in C_{2 \pi}$. Then we have

$$
\sup _{t \in \mathbb{R}} z(t)=\sup _{t \in \mathbb{R}} z(t-\mu(t)) .
$$

Proof. It is obvious that

$$
\sup _{t \in \mathbb{R}} z(t-\mu(t)) \leq \sup _{t \in \mathbb{R}} z(t)=z\left(t_{0}\right),
$$

and because $z(t) \in C_{2 \pi}$, there exists $t_{0} \in \mathbb{R}$ such that $\sup _{t \in \mathbb{R}} z(t)=z\left(t_{0}\right)$. Note that $t-\mu(t)$ maps $\mathbb{R}$ onto $\mathbb{R}$. Hence, there exists $t_{1} \in \mathbb{R}$ such that

$$
z\left(t_{1}-\mu\left(t_{1}\right)\right)=z\left(t_{0}\right) .
$$

Together with (3.6) and (3.7), this implies that (3.5) holds. 
From Lemma 3.1 we deduce that

$$
\|T x-T y\|=|a| \sup _{t \in \mathbb{R}}|x(t-\tau)-y(t-\tau)|=|a|\|x-y\| .
$$

The above expression shows that $T: C_{2 \pi} \rightarrow C_{2 \pi}$ is contractive if $|a|<1$, is isometric if $|a|=1$, and is expansive if $|a|>1$. The case of $|a| \neq 1$ is not very hard since it may be solved by the existing Krasnosel'skii fixed point theorems. For our purpose, we are mainly concerned with the critical case when $|a|=1$.

Let $C_{2 \pi}^{1}=C^{1}(\mathbb{R}, \mathbb{R}) \cap C_{2 \pi}$ and let $D(T)=\left\{x: x \in C_{2 \pi}\right.$ and $\left.(I-T) x \in C_{2 \pi}^{1}\right\}$. Then $D(T) \subset C_{2 \pi}$, and we have the following key lemma.

Lemma 3.3. Assume that $a=-1$ and $\tau=\frac{2 \pi}{3}$. Then $I-T$ maps $D(T)$ onto $C_{2 \pi}^{1}$ and $(I-T) x_{n} \rightarrow y$ implies that $\left\{x_{n_{k}}\right\}$ is convergent, where $\left\{x_{n_{k}}\right\}$ is a subsequence of $\left\{x_{n}\right\}$.

Proof. Let

$$
L_{2 \pi}^{2}=\left\{u \mid u(t)=u(t+2 \pi) \text { for a.e. } t \in \mathbb{R} \text { and } \int_{0}^{2 \pi}|u(t)|^{2} d t<\infty\right\}
$$

be a Banach space with the norm $\|u\|_{2}=\left(\int_{0}^{2 \pi}|u(t)|^{2} d t\right)^{1 / 2}$. Then it is well known that

$$
C_{2 \pi}, \quad L_{2 \pi}^{2} \subset \overline{\operatorname{span}}\{1, \quad \cos k t, \quad \sin k t \mid \quad k \in \mathbb{Z}\} .
$$

We perform the calculations by expanding $u \in C_{2 \pi}$ in a Fourier series with respect to the complete orthonormal system. For each $u \in C_{2 \pi}^{1}$, we then have $u^{\prime} \in C_{2 \pi}$ and so

$$
u(t)=\sum_{k=-\infty}^{\infty} u_{k} e^{i k t}, \quad u^{\prime}(t)=\sum_{k=-\infty}^{\infty} u_{k}^{\prime} e^{i k t}
$$

where

$$
u_{k}=\frac{1}{2 \pi} \int_{0}^{2 \pi} u(s) e^{-i k s} d s, \quad u_{k}^{\prime}=\frac{1}{2 \pi} \int_{0}^{2 \pi} u^{\prime}(s) e^{-i k s} d s, \quad k \in \mathbb{Z} .
$$

Consequently,

$$
u_{k}^{\prime}=\frac{1}{2 \pi} \int_{0}^{2 \pi} u^{\prime}(s) e^{-i k s} d s=\frac{1}{2 \pi} \int_{0}^{2 \pi} e^{-i k s} d u(s)=\frac{i k}{2 \pi} \int_{0}^{2 \pi} u(s) e^{-i k s} d s=i k u_{k} .
$$

If one puts

$$
v(t)=\frac{u_{0}}{2}+\sum_{k \neq 0} \frac{u_{k}}{1+e^{-2 \pi i k / 3}} e^{i k t}
$$

by a direct calculation, one then derives $(I-T) v(t)=u(t)$. From (3.8) and (3.9), one can easily deduce that the Fourier series of $v^{\prime}$ is

$$
\sum_{k \neq 0} \frac{i k u_{k}}{1+e^{-2 \pi i k / 3}} e^{i k t}
$$

It is straightforward to see that

$$
\inf _{k \in \mathbb{Z}}\left\{\cos \frac{2 \pi}{3} k\right\}=\min \left\{1, \cos \frac{2 \pi}{3}, \cos \frac{4 \pi}{3}\right\}=-\frac{1}{2} .
$$


We therefore obtain

$$
\inf _{k \in \mathbb{Z}}\left|1+e^{-\frac{2 \pi i k}{3}}\right|=\inf _{k \in \mathbb{Z}} \sqrt{2\left(1+\cos \frac{2 \pi}{3} k\right)}=1 .
$$

The equalities (3.8) and (3.10) give us that

$$
\sum_{k \neq 0}\left|\frac{u_{k}}{1+e^{-2 \pi i k / 3}}\right| \leq \sum_{k \neq 0}\left|u_{k}\right|=\sum_{k \neq 0}\left|\frac{u_{k}^{\prime}}{i k}\right| \leq\left(\sum_{k \neq 0}\left|u_{k}^{\prime}\right|^{2}\right)^{1 / 2}\left(\sum_{k \neq 0} \frac{1}{|k|^{2}}\right)^{1 / 2}=\sqrt{\frac{\pi}{6}}\left\|u^{\prime}\right\|_{2}
$$

and

$$
\left(\sum_{k \neq 0}\left|\frac{i k u_{k}}{1+e^{-2 \pi i k / 3}}\right|^{2}\right)^{1 / 2} \leq\left(\sum_{k \neq 0}\left|u_{k}^{\prime}\right|^{2}\right)^{1 / 2}=\frac{1}{\sqrt{2 \pi}}\left\|u^{\prime}\right\|_{2}<\infty .
$$

These estimates show that $v \in C_{2 \pi}$ and

$$
\left\|v-\frac{u_{0}}{2}\right\| \leq \sqrt{\frac{\pi}{6}}\left\|u^{\prime}\right\|_{2} .
$$

Moreover $v^{\prime} \in L_{2 \pi}^{2}$, and by employing Parseval's inequality,

$$
\|v\|_{2}=\sqrt{2 \pi}\left(\sum_{k \neq 0}\left|\frac{i k u_{k}}{1+e^{-2 \pi i k / 3}}\right|^{2}\right)^{1 / 2} \leq\left\|u^{\prime}\right\|_{2}
$$

and thus $v \in D(T)$. Suppose now that $\left\{v_{n}\right\} \subset D(T)$ with $(I-T) v_{n} \rightarrow u$ in $C_{2 \pi}$. Putting $u_{n}=(I-T) v_{n}$, then $u_{n} \rightarrow u$ in $C_{2 \pi}$ and for each $k \in \mathbb{Z}$,

$$
u_{k}^{(n)}=\frac{1}{2 \pi} \int_{0}^{2 \pi} u_{n}(s) e^{-i k s} d s \rightarrow \frac{1}{2 \pi} \int_{0}^{2 \pi} u(s) e^{-i k s} d s=u_{k}, \quad \text { as } n \rightarrow \infty .
$$

Accordingly, one can infer from (3.9) and (3.11) that

$$
v_{n}(t)=\frac{u_{0}^{(n)}}{2}+\sum_{k \neq 0} \frac{u_{k}^{(n)}}{1+e^{-2 \pi i k / 3}} e^{i k t} \rightarrow \frac{u_{0}}{2}+\sum_{k \neq 0} \frac{u_{k}}{1+e^{-2 \pi i k / 3}} e^{i k t} .
$$

This concludes the proof of the lemma.

Lemma 3.4. The operator $S$ maps bounded sets of $C_{2 \pi}$ into relatively compact sets.

Proof. It has been shown that $(S y)(t+2 \pi)=(S y)(t)$ for all $t \in \mathbb{R}$. It is obvious from the definition of $S$ that $S y$ is continuous and hence $S y \in C_{2 \pi}$.

Let $B$ be a bounded subset of $C_{2 \pi}$ with $\|y\| \leq R$ for all $y \in B$, where $R>0$ is a constant. For $t \in[0,2 \pi]$, which implies that $s \in[t, t+2 \pi] \subseteq[0,4 \pi]$, and for $y \in B$ we have by the expression of $S$ that

$$
|(S y)(t)| \leq \int_{t}^{t+2 \pi}|G(t, s) f(s, y(s-\tau))-\operatorname{ar}(s) y(s-\tau)| d s \leq G_{2 \pi} f_{2 \pi, R},
$$

where $f_{2 \pi, R}=\max \{|f(s, u)-r(s) u|:(s, u) \in[0,4 \pi] \times[-R, R]\}$ and $G_{2 \pi}=$ $\max \left\{\int_{t}^{t+2 \pi}|G(t, s)| d s: 0 \leq t \leq 2 \pi\right\}$. The above estimate illustrates that $\|S y\| \leq$ $G_{2 \pi} f_{2 \pi, R}$, which shows that $S(B)$ is uniformly bounded. Next, let $y \in B$. Then a differentiation of $(S y)(t)$ with respect to $t$ yields

$$
(S y)^{\prime}(t)=-[f(t, y(t-\tau))-\operatorname{ar}(t) y(t-\tau)]+r(t)(S y)(t) .
$$

Hence, by taking the supremum norm we get

$$
\left\|(S y)^{\prime}\right\| \leq\left(1+G_{2 \pi}\|r\|\right) f_{2 \pi, R} .
$$


This inequality implies that $S(B)$ is an equicontinuous subset of $C_{2 \pi}$, Then using the Arzelà-Ascoli Theorem we obtain that $S$ is a compact mapping. In the light of the supremum norm and the continuity of all items in $S$, it is an easy matter to verify that $S$ is continuous. Therefore $S: C_{2 \pi} \rightarrow C_{2 \pi}$ is completely continuous. This proves the lemma.

Theorem 3.1. Let $a=-1, \tau=\frac{2 \pi}{3}, \bar{r} \neq 0$, and let $K \subset D(T)$ be a bounded, closed and convex subset of the Banach space $\left(C_{2 \pi},\|\cdot\|\right)$. Assume that for each $y \in K$ the solution $x$ of

$$
x(t)=a x(t-\tau)+\int_{t}^{t+2 \pi} G(t, s)[f(s, y(s-\tau))-r(s) y(s-\tau)] d s
$$

resides in $K$. Then the equation (3.1) admits one continuous $2 \pi$-periodic solution in $K$.

Proof. It follows from Lemma 3.1 and the definitions of $S$ and $T$ that the equation (3.1) is equivalent to the fixed point problem

$$
x=T x+S x .
$$

Since $S y \in C_{2 \pi}^{1}$ for each $y \in C_{2 \pi}$, Lemma 3.3 gives $S(K) \subset(I-T)(D(T)) \subset(I-$ $T)\left(C_{2 \pi}\right)$. This in conjunction with (3.12) guarantees that the set $\mathcal{F}\left(C_{2 \pi}, K ; T, S\right)$ is nonempty and that the conditions (i) and (iii) of Theorem 2.3 are satisfied. Notice that $T: C_{2 \pi} \rightarrow C_{2 \pi}$ is bounded and linear and the fourth observation of Remark 2.2. Then the verifications of the assumptions (iv) and (v) are trivial. Lemma 3.4, coupled with the second paragraph of Remark 2.3, illustrates that the set $\mathcal{F}$ is relatively compact.

Consequently, by using Theorem 2.2 or Theorem 2.3. one knows that (3.13) admits a solution in $K$; that is, the equation (3.1) has at least one $2 \pi$-periodic solution in $K$.

By imposing a growth condition on $f$, we will show that the assumption (3.12) is satisfied. Furthermore the boundedness of $K$ is not required. To this end, we ask that $f$ fulfills

$$
f_{4 \pi, \mathbb{R}}=\sup \{|f(s, u)-\operatorname{ar}(s) u|:(s, u) \in[0,4 \pi] \times \mathbb{R}\}<\infty .
$$

Corollary 3.1. Let $a=-1, \tau=\frac{2 \pi}{3}$ and $\bar{r} \neq 0$. Assume that $f$ satisfies the growth condition (3.14). Then the equation (3.1) has at least one continuous $2 \pi$-periodic solution.

Proof. Keeping (3.14) in mind, using essentially the same arguments as in Lemma 3.4, one can readily conclude that $S\left(C_{2 \pi}\right)$ is a relatively compact subset of $C_{2 \pi}$. Recall that $D(T)=\left\{x: x \in C_{2 \pi}\right.$ and $\left.(I-T) x \in C_{2 \pi}^{1}\right\}$. So $D(T)$ is a linear subspace of $C_{2 \pi}$, but it may not be closed. Accordingly, $\overline{D(T)}$ is a closed convex subset of $C_{2 \pi}$. Lemma 3.3 now implies that (3.12) is satisfied on the set $\overline{D(T)}$. The conclusion of the lemma follows in a similar way to Theorem 3.1 . 


\section{REFERENCES}

[1] R. R. Akhmerov, M. I. Kamenskii, A. S. Potapov, A. E. Rodkina and B. N. Sadovskii, Measures of Noncompactness and Condensing Operators. Birkhäuser, Basel (1992). MR 1153247 (92k:47104)

[2] J. Banas, K. Goebel, Measures of Noncompactness in Banach Spaces, Marcel Dekker, New York (1980). MR591679 (82f:47066)

[3] E. Beretta, F. Solimano, Y. Takeuchi, A mathematical model for drug administration by using the phagocytosis of red blood cells, J. Math. Biol. 35 (1996), 1-19. MR1478581 (99g:92014)

[4] T. Burton, A fixed-point theorem of Krasnosel'skii, Appl. Math. Lett., 11 (1998), 85-88. MR 1490385 (98i:47053)

[5] C. S. Barroso, E.V. Teixeira, A topological and geometric approach to fixed points results for sum of operators and applications, Nonlinear Anal. 60 (2005), 625-650. MR2109150 (2005i:47087)

[6] S. N. Chow, Existence of periodic solutions of autonomous functional differential equations, J. Diff. Eqns. 15 (1974), 350-378. MR0336003 (49:779)

[7] B. C. Dhage, Remarks on two fixed-point theorems involving the sum and the product of two operators, Comput. Math. Appl. 46 (2003), 1779-1785. MR2018766 (2004i:47104)

[8] D. Henry, Geometric Theory of Semilinear Parabolic Equations, Springer-Verlag, Berlin-New York, 1981. MR610244 (83j:35084)

[9] M. A. Krasnosel'skii, Two remarks on the method of successive approximations, Uspehi Mat. Nauk 10 (1955), 123-127. MR0068119 (16:833a)

[10] Y. K. Li, Y. Kuang, Periodic solutions of periodic delay Lotka-Volterra equations and systems, J. Math. Anal. Appl. 255 (2001), 260-280. MR.1813821 (2001k:34133)

[11] Y. C. Liu and Z. X. Li, Krasnoselskii type fixed point theorems and applications, Proc. Amer. Math. Soc. 136 (2008), 1213-1220. MR2367095 (2008k:47113)

[12] E. A. Ok, Fixed set theorems of Krasnoselskii type, Proc. Amer. Math. Soc. 137 (2009), 511-518. MR2448571 (2009f:47099)

[13] J. W. H. So, J. Wu, X. Zou, Structured population on two patches: Modeling dispersal and delay, J. Math. Biol. 43 (2001), 37-51. MR.1854000 (2003b:92023)

[14] S. Park, Generalizations of the Krasnoselskii fixed point theorem, Nonlinear Anal. 67 (2007), 3401-3410. MR2350896(2009a:47111)

[15] J. Wu, Theory and Application of Partial Functional Differential Equations, Springer-Verlag, Berlin-New York, 1996. MR,1415838 (98a:35135)

[16] T. Xiang and R. Yuan, A class of expansive-type Krasnoselskii fixed point theorem, Nonlinear Anal. 71 (2009), 3229-3239. MR 2532845

[17] E. Zeidler, Nonlinear Functional Analysis and its Applications, I: Fixed Point Theorems, Springer-Verlag, New York, 1986. MR816732 (87f:47083)

Laboratory of Mathematics and Complex Systems, School of Mathematical Sciences, Beijing Normal University, Ministry of Education, Beijing 100875, People's Republic of CHINA

E-mail address: tianx@mail.bnu.edu.cn

Laboratory of Mathematics and Complex Systems, School of Mathematical Sciences, Beijing Normal University, Ministry of Education, Beijing 100875, People's Republic of CHINA

E-mail address: ryuan@bnu.edu.cn 